\title{
The Existence, Uniqueness, and Stability Analysis of the Discrete Fractional Three-Point Boundary Value Problem for the Elastic Beam Equation
}

\author{
Jehad Alzabut ${ }^{1,2, *,+}+\mathbb{D}$, A. George Maria Selvam ${ }^{3,+} \mathbb{D}^{\mathbb{D}}$, R. Dhineshbabu $^{4,+} \mathbb{D}^{\mathbb{D}}$ and Mohammed K. A. Kaabar ${ }^{5,6, *, t}(\mathbb{D}$ \\ 1 Department of Mathematics and General Sciences, Prince Sultan University, Riyadh 11586, Saudi Arabia \\ Group of mathematics, Faculty of Engineering, Ostim Technical University, Ankara 06374, Turkey \\ 3 Department of Mathematics, Sacred Heart College (Autonomous), Tirupattur 635 601, Tamil Nadu, India; \\ agms@shctpt.edu \\ 4 Department of Mathematics, Sri Venkateswara College of Engineering and Technology (Autonomous), \\ Chittoor 517 127, Andhra Pradesh, India; dhineshbabur@svcetedu.org \\ 5 Department of Mathematics and Statistics, Washington State University, Pullman, WA 99163, USA \\ 6 Institute of Mathematical Sciences, Faculty of Science, University of Malaya, \\ Kuala Lumpur 50603, Malaysia \\ * Correspondence: jalzabut@psu.edu.sa (J.A.); mohammed.kaabar@wsu.edu (M.K.A.K.); \\ Tel.: +966-114948547 (J.A.) \\ $+\quad$ These authors contributed equally to this work.
}

check for

updates

Citation: Alzabut, J.; Selvam, A.G.; Dhineshbabu, R.; Kaabar, M.K.A. The Existence, Uniqueness, and Stability Analysis of the Discrete Fractional Three-Point Boundary Value Problem for the Elastic Beam Equation. Symmetry 2021, 13, 789. https:// doi.org/10.3390/sym13050789

Academic Editor: Clemente Cesarano

Received: 27 February 2021

Accepted: 29 April 2021

Published: 2 May 2021

Publisher's Note: MDPI stays neutral with regard to jurisdictional claims in published maps and institutional affiliations.

Copyright: (C) 2021 by the authors. Licensee MDPI, Basel, Switzerland. This article is an open access article distributed under the terms and conditions of the Creative Commons Attribution (CC BY) license (https:/ / creativecommons.org/licenses/by/ $4.0 /)$.

\begin{abstract}
An elastic beam equation (EBEq) described by a fourth-order fractional difference equation is proposed in this work with three-point boundary conditions involving the Riemann-Liouville fractional difference operator. New sufficient conditions ensuring the solutions' existence and uniqueness of the proposed problem are established. The findings are obtained by employing properties of discrete fractional equations, Banach contraction, and Brouwer fixed-point theorems. Further, we discuss our problem's results concerning $\mathcal{H}$ yers- $\mathcal{U}$ lam $(\mathcal{H U})$, generalized $\mathcal{H}$ yers- $\mathcal{U}$ lam $(\mathcal{G H \mathcal { U }})$, $\mathcal{H}$ yers- $\mathcal{U}$ lam- $\mathcal{R}$ assias $(\mathcal{H} \mathcal{U} \mathcal{R})$, and generalized $\mathcal{H}$ yers- $\mathcal{U}$ lam- $\mathcal{R}$ assias $(\mathcal{G} \mathcal{H} \mathcal{U} \mathcal{R})$ stability. Specific examples with graphs and numerical experiment are presented to demonstrate the effectiveness of our results.
\end{abstract}

Keywords: Riemann-Liouville fractional difference operator; boundary value problem; discrete fractional calculus; existence and uniqueness; Ulam stability; elastic beam problem

MSC: 34A12; 34B10; 34B15; 39A12; 47H10; 74B20

\section{Introduction}

Elastic beam (EB) deflections are commonly known phenomena in science and engineering. Based on the significance of their applications such as for aircraft design, chemical sensors, micro-electromechanical systems, material mechanics, medical diagnostics, and physics, two-point boundary value problems (BVPs) for EBEqs have received considerable attention. Recently, many researchers have investigated EBEqs with various boundary conditions (BCs) (refer to [1-6]). Gupta in [6] studied a fourth-order EBEq with two-point BCs:

$$
\left\{\begin{array}{l}
w^{(4)}(\kappa)=G(\kappa, w(\kappa)), \kappa \in(0,1) \\
w(0)=0, w^{\prime \prime}(0)=0, w^{\prime}(1)=0, w^{\prime \prime \prime}(1)=0
\end{array}\right.
$$

Equation (1) describes an elastic beam model of length 1, which is clamped with a displacement and a bending moment that are equal to zero at the left end, and this model is free to travel with disappearing angular attitude and shear force at the right end. 
In addition, Cianciaruso et al. [1] studied the model of the cantilever beam equation with three-point BCs:

$$
\left\{\begin{array}{l}
w^{(4)}(\kappa)=G(\kappa, w(\kappa)), \kappa \in(0,1) \\
w(0)=w^{\prime}(0)=w^{\prime \prime}(1)=0, w^{\prime \prime \prime}(1)=h(w(\zeta))
\end{array}\right.
$$

where $\zeta \in(0,1)$ is a real constant. The above is a feedback mechanism model where the shearing force at the beam's right end responds to the displacement experienced at a point $\zeta$.

Fractional calculus (FC) is a generalized form of classical integer-order calculus. Fractional calculus examines the properties of fractional-order derivatives and integrals. Due to its numerous applications in various scientific fields, this research area has gained considerable attention over the past few years. FC can be applicable in several fields of science and engineering, along with aerodynamics, electrical circuits, fluid dynamics, heat conduction, and physics. We refer to the comprehensive works in [7-10] for a detailed analysis of its applications, and we refer to [11-15] for the latest trends in the area of FC.

Researchers have explored various aspects of fractional difference equations (FDEs). Obviously, the solutions' existence, uniqueness, and stability analysis are some important features of FDEs. Various analytical approaches and fixed-point theory have been used to examine the solutions' existence and stability for FDEs. Several researchers have contributed a number of books and papers in this regard [16]. However, finding the exact solution of nonlinear FDEs is often too difficult; therefore, the stability analysis of solutions plays a crucial role in such investigations. Various kinds of stabilities described in the past are discussed in the literature, such as Lyapunov stability [17], Mittag-Leffler stability [18], and exponential stability [19]. Presumably, the most dependable stabilities are called $\mathcal{H U}$ stability. The discussed stability was modified to $\mathcal{G H \mathcal { H }}$ stability (refer to [20-22]). In 1970, Rassias further generalized the aforesaid stability. For FDEs with different BCs concerning Riemann-Liouville and Caputo operators, the addressed fields of existence and stability analysis are well-equipped (see [23-28]).

A new interesting research field, named discrete fractional calculus (DFC), is attracting the interest of mathematicians and researchers. With discrete fractional operators, several real-world problems are being investigated [29-32]. The fractional difference equations have recently become an interesting field for scientists because of their applications in biology, ecology, and applied sciences [33]. However, a few research studies that have been conducted on discrete fractional-order BVPs can be found in [34-47].

The above findings inspired us in this study concerning the solutions' existence and uniqueness with various types of Ulam stability results for the proposed discrete fractional elastic beam equation (FEBE) that is subject to the three-point BCs as follows:

$$
\left\{\begin{array}{l}
\Delta_{\beta-4}^{\beta} w(\kappa)=G(\kappa+\beta-1, w(\kappa+\beta-1)), \kappa \in \mathbb{N}_{0}^{n+3} \\
w(\beta-4)=0, \Delta^{2} w(\beta-4)=0, \Delta w(\beta+n)=0, \Delta^{3} w(\beta+n)+w(\zeta)=0
\end{array}\right.
$$

where $\beta \in(3,4]$ is a fractional order and $\zeta \in \mathbb{N}_{\beta-1}^{\beta+n+2}$ is constant. Here, we have that $G: \mathbb{N}_{\beta-4}^{\beta+n+3} \times \mathbb{R} \rightarrow \mathbb{R}$ is continuous, $w: \mathbb{N}_{\beta-4}^{\beta+n+3} \rightarrow \mathbb{R}, \Delta_{\beta-4}^{\beta}$ is the Riemann-Liouville discrete fractional operator, and $n \in \mathbb{N}_{0}$.

The rest of this research work is structured as follows. Basic background knowledge on DFC is stated in Section 2. The result for a linear version of the BVP Equation (2) is discussed in Section 3. Further, by using this solution, the existence and uniqueness conditions for the proposed discrete FEBE with three-point BCs (Equation (2)) are derived with the help of contraction mapping and the Brouwer fixed-point theorems. Different types of stability results are extensively obtained in Section 4 via the findings of nonlinear analysis. Some illustrative examples with graphs and numerical experiment are presented in Section 5 as applications to provide a better understanding of our findings. Finally, Section 6 concludes our research work. 


\section{Essential Preliminaries}

Some important notions and preliminary lemmas are stated in this section, which are needed for discussion of our results.

Definition 1 ([30]). For $\beta>0$, the $\beta$ th order fractional sum of $G$ can be defined as

$$
\Delta^{-\beta} G(\kappa)=\frac{1}{\Gamma(\beta)} \sum_{i=a}^{\kappa-\beta}(\kappa-\sigma(i))^{(\beta-1)} G(i),
$$

for $\kappa \in \mathbb{N}_{a+\beta}$ and $\sigma(i)=i+1$. Define the $\beta$ th fractional difference for $\beta>0$ by $\Delta^{\beta} G(\kappa):=$ $\Delta^{M} \Delta^{\beta-M} G(\kappa)$, for $\kappa \in \mathbb{N}_{a+M-\beta}, M \in \mathbb{N}$ satisfies $0 \leq M-1<\beta \leq M$, and $\kappa^{(\beta)}:=$ $\frac{\Gamma(\kappa+1)}{\Gamma(\kappa+1-\beta)}$.

Lemma 1 ([30]). Assume that $\kappa$ and $\beta$ are any numbers such that $\kappa^{(\beta)}$ and $\kappa^{(\beta-1)}$ are defined. Then we have $\Delta \kappa^{(\beta)}=\beta \kappa^{(\beta-1)}$.

Lemma 2 (see $[34,44])$. Let $0 \leq M-1<\beta \leq M$. Then,

$$
\Delta^{-\beta} \Delta^{\beta} G(\kappa)=G(\kappa)+\mathcal{C}_{1} \kappa^{(\beta-1)}+\mathcal{C}_{2} \kappa^{(\beta-2)}+\ldots+\mathcal{C}_{M} \mathcal{\kappa}^{(\beta-M)},
$$

for some $\mathcal{C}_{j} \in \mathbb{R}, 1 \leq j \leq M$

Lemma 3 (see [42]). For $\kappa$ and $i$, for which both $(\kappa-\sigma(i))^{(\beta)}$ and $(\kappa-1-\sigma(i))^{(\beta)}$ are defined, we obtain that $\Delta_{i}\left[(\kappa-\sigma(i))^{(\beta)}\right]=-\beta(\kappa-1-\sigma(i))^{(\beta-1)}$.

Lemma 4 (see $[43,46])$. Let $\beta, v>0$. Then,

$$
\Delta^{-\beta} \kappa^{(v)}=\frac{\Gamma(v+1)}{\Gamma(\nu+\beta+1)} \kappa^{(v+\beta)} \text { and } \Delta^{\beta} \kappa^{(v)}=\frac{\Gamma(v+1)}{\Gamma(v-\beta+1)} \kappa^{(v-\beta)} .
$$

\section{EB Existence and Uniqueness}

The existence and uniqueness of EB is established in this section to the three-point BCs for the proposed discrete FEBE Equation (2). We now introduce the following theorem that deals with a linear variant solution of our proposed BVP Equation (2).

Theorem 1. Let $H: \mathbb{N}_{\beta-4}^{\beta+n+3} \rightarrow \mathbb{R}$ be given. Then, the linear discrete FEBE with three-point BCs:

$$
\left\{\begin{array}{l}
\Delta_{\beta-4}^{\beta} w(\kappa)=H(\kappa+\beta-1), \kappa \in \mathbb{N}_{0}^{n+3} \\
w(\beta-4)=0, \Delta^{2} w(\beta-4)=0, \Delta w(\beta+n)=0, \Delta^{3} w(\beta+n)+w(\zeta)=0
\end{array}\right.
$$

has the unique solution, for $\kappa \in \mathbb{N}_{\beta-4}^{\beta+n+3}$,

$$
\begin{aligned}
w(\kappa)= & \frac{1}{\Gamma(\beta)} \sum_{i=0}^{\kappa-\beta}(\kappa-\sigma(i))^{(\beta-1)} H(i+\beta-1) \\
& +\mathbb{E}_{1}(\kappa)\left[\frac{1}{\Gamma(\beta)} \sum_{i=0}^{\zeta-\beta}(\zeta-\sigma(i))^{(\beta-1)}+\frac{1}{\Gamma(\beta-3)} \sum_{i=0}^{n+3}(\beta+n-\sigma(i))^{(\beta-4)}\right] H(i+\beta-1) \\
& +\frac{\mathbb{E}_{2}(\kappa)}{\Gamma(\beta-1)} \sum_{i=0}^{n+1}(\beta+n-\sigma(i))^{(\beta-2)} H(i+\beta-1),
\end{aligned}
$$


where

$$
\mathbb{E}_{1}(\kappa)=\frac{\left[{\frac{\kappa^{(\beta-1)}}{e_{1}}}^{\prime} h_{1}+\kappa^{(\beta-2)} f_{1} f_{4}-\kappa^{(\beta-3)} f_{1}\right]}{K} ; \mathbb{E}_{2}(\kappa)=\frac{\left[\kappa^{(\beta-1)} h_{2}-\kappa^{(\beta-2)} e_{1} f_{4}+\kappa^{(\beta-3)} e_{1}\right]}{K}
$$

such that $h_{1}=f_{1}\left(e_{3}-e_{2} f_{4}\right)-K, h_{2}=e_{2} f_{4}-e_{3}, K=\left[e_{3} f_{1}-e_{1} f_{3}\right]-f_{4}\left[e_{2} f_{1}-e_{1} f_{2}\right]$, $e_{1}=(\beta-1)^{(3)}(\beta+n)^{(\beta-4)}+\zeta^{(\beta-1)}, e_{2}=(\beta-2)^{(3)}(\beta+n)^{(\beta-5)}+\zeta^{(\beta-2)}$, $e_{3}=(\beta-3)^{(3)}(\beta+n)^{(\beta-6)}+\zeta^{(\beta-3)}, f_{1}=(\beta-1)(\beta+n)^{(\beta-2)}$, $f_{2}=(\beta-2)(\beta+n)^{(\beta-3)}, f_{3}=(\beta-3)(\beta+n)^{(\beta-4)}$ and $f_{4}=\frac{(\beta-4)}{(\beta-2)}$.

Proof. By applying the fractional sum $\Delta^{-\beta}$ of order $\beta \in(3,4]$ along with Lemma 2 to Equation (3), we have

$$
w(\kappa)=\frac{1}{\Gamma(\beta)} \sum_{i=0}^{\kappa-\beta}(\kappa-\sigma(i))^{(\beta-1)} H(i+\beta-1)+\mathcal{C}_{1} \kappa^{(\beta-1)}+\mathcal{C}_{2} \kappa^{(\beta-2)}+\mathcal{C}_{3} \kappa^{(\beta-3)}+\mathcal{C}_{4} \kappa^{(\beta-4)},
$$

for $\kappa \in \mathbb{N}_{\beta-4}^{\beta+n+3}$ and some constants $\mathcal{C}_{j} \in \mathbb{R}$, where $j=1,2,3$, 4. By applying the first BC $w(\beta-4)=0$ in Equation (6), we obtain

$w(\beta-4)=\mathcal{C}_{1}(\beta-4)^{(\beta-1)}+\mathcal{C}_{2}(\beta-4)^{(\beta-2)}+\mathcal{C}_{3}(\beta-4)^{(\beta-3)}+\mathcal{C}_{4}(\beta-4)^{(\beta-4)}=0$.

By using Definition 1, we obtain

$$
(\beta-4)^{(\beta-1)}=(\beta-4)^{(\beta-2)}=(\beta-4)^{(\beta-3)}=0 \text { and }(\beta-4)^{(\beta-4)}=\Gamma(\beta-3) .
$$

Equations (7) and (8) imply $\mathcal{C}_{4}=0$. Using $\mathcal{C}_{4}$ in Equation (6) provides

$$
w(\kappa)=\frac{1}{\Gamma(\beta)} \sum_{i=0}^{\kappa-\beta}(\kappa-\sigma(i))^{(\beta-1)} H(i+\beta-1)+\mathcal{C}_{1} \kappa^{(\beta-1)}+\mathcal{C}_{2} \kappa^{(\beta-2)}+\mathcal{C}_{3} \kappa^{(\beta-3)} .
$$

Using Lemma 1 and taking the operator $\Delta$ on both sides of Equation (9), we obtain

$$
\begin{aligned}
\Delta w(\kappa)= & \frac{1}{\Gamma(\beta-1)} \sum_{i=0}^{\kappa-\beta+1}(\kappa-\sigma(i))^{(\beta-2)} H(i+\beta-1) \\
& +\mathcal{C}_{1}(\beta-1) \kappa^{(\beta-2)}+\mathcal{C}_{2}(\beta-2) \kappa^{(\beta-3)}+\mathcal{C}_{3}(\beta-3) \kappa^{(\beta-4)} .
\end{aligned}
$$

From the third BC $\Delta w(\beta+n)=0$ in Equation (10), we obtain

$$
\frac{1}{\Gamma(\beta-1)} \sum_{i=0}^{n+1}(\beta+n-\sigma(i))^{(\beta-2)} H(i+\beta-1)+\mathcal{C}_{1} f_{1}+\mathcal{C}_{2} f_{2}+\mathcal{C}_{3} f_{3}=0 .
$$

The operator $\Delta$ is applied on both sides of Equation (10) with the aid of Lemma 1, and we obtain

$$
\begin{aligned}
\Delta^{2} w(\kappa)= & \frac{1}{\Gamma(\beta-2)} \sum_{i=0}^{\kappa-\beta+2}(\kappa-\sigma(i))^{(\beta-3)} H(i+\beta-1)+\mathcal{C}_{1}(\beta-1)^{(2)} \kappa^{(\beta-3)} \\
& +\mathcal{C}_{2}(\beta-2)^{(2)} \kappa^{(\beta-4)}+\mathcal{C}_{3}(\beta-3)^{(2)} \mathcal{K}^{(\beta-5)}
\end{aligned}
$$

The second BC of Equation (3) implies

$$
\mathcal{C}_{2}(\beta-2)+\mathcal{C}_{3}(\beta-4)=0 .
$$


Again, using Lemma 1 and taking the operator $\Delta$ on both sides of Equation (12), we obtain

$$
\begin{aligned}
\Delta^{3} w(\kappa)= & \frac{1}{\Gamma(\beta-3)} \sum_{i=0}^{\kappa-\beta+3}(\kappa-\sigma(i))^{(\beta-4)} H(i+\beta-1)+\mathcal{C}_{1}(\beta-1)^{(3)} \kappa^{(\beta-4)} \\
& +\mathcal{C}_{2}(\beta-2)^{(3)} \kappa^{(\beta-5)}+\mathcal{C}_{3}(\beta-3)^{(3)} \mathcal{K}^{(\beta-6)}
\end{aligned}
$$

Using the last $\mathrm{BC} \Delta^{3} w(\beta+n)+w(\zeta)=0$ in Equations (9) and (14) yields

$$
w(\zeta)=\frac{1}{\Gamma(\beta)} \sum_{i=0}^{\zeta-\beta}(\zeta-\sigma(i))^{(\beta-1)} H(i+\beta-1)+\mathcal{C}_{1} \zeta^{(\beta-1)}+\mathcal{C}_{2} \zeta^{(\beta-2)}+\mathcal{C}_{3} \zeta^{(\beta-3)}
$$

and

$$
\begin{aligned}
\Delta^{3} w(\beta+n)= & \frac{1}{\Gamma(\beta-3)} \sum_{i=0}^{n+3}(\beta+n-\sigma(i))^{(\beta-4)} H(i+\beta-1)+\mathcal{C}_{1}(\beta-1)^{(3)}(\beta+n)^{(\beta-4)} \\
& +\mathcal{C}_{2}(\beta-2)^{(3)}(\beta+n)^{(\beta-5)}+\mathcal{C}_{3}(\beta-3)^{(3)}(\beta+n)^{(\beta-6)} .
\end{aligned}
$$

From Equations (15) and (16), and by employing the last BC Equation (3), we obtain

$$
\begin{aligned}
& \frac{1}{\Gamma(\beta-3)} \sum_{i=0}^{n+3}(\beta+n-\sigma(i))^{(\beta-4)} H(i+\beta-1) \\
& +\frac{1}{\Gamma(\beta)} \sum_{i=0}^{\zeta-\beta}(\zeta-\sigma(i))^{(\beta-1)} H(i+\beta-1)+\mathcal{C}_{1} e_{1}+\mathcal{C}_{2} e_{2}+\mathcal{C}_{3} e_{3}=0
\end{aligned}
$$

Solving Equations (11) and (17), we obtain

$$
\begin{aligned}
& f_{1}\left(\frac{1}{\Gamma(\beta-3)} \sum_{i=0}^{n+3}(\beta+n-\sigma(i))^{(\beta-4)}+\frac{1}{\Gamma(\beta)} \sum_{i=0}^{\zeta-\beta}(\zeta-\sigma(i))^{(\beta-1)}\right) H(i+\beta-1) \\
& +\mathcal{C}_{2}\left(e_{2} f_{1}-e_{1} f_{2}\right)+\mathcal{C}_{3}\left(e_{3} f_{1}-e_{1} f_{3}\right)-\frac{e_{1}}{\Gamma(\beta-1)} \sum_{i=0}^{n+1}(\beta+n-\sigma(i))^{(\beta-2)} H(i+\beta-1)=0 .
\end{aligned}
$$

Now, a constant $\mathcal{C}_{3}$ is found by solving Equations (13) and (18) as follows:

$$
\begin{aligned}
\mathcal{C}_{3} & =\frac{1}{K}\left[\frac{e_{1}}{\Gamma(\beta-1)} \sum_{i=0}^{n+1}(\beta+n-\sigma(i))^{(\beta-2)} H(i+\beta-1)\right. \\
& \left.-f_{1}\left(\frac{1}{\Gamma(\beta)} \sum_{i=0}^{\zeta-\beta}(\zeta-\sigma(i))^{(\beta-1)}+\frac{1}{\Gamma(\beta-3)} \sum_{i=0}^{n+3}(\beta+n-\sigma(i))^{(\beta-4)}\right) H(i+\beta-1)\right] .
\end{aligned}
$$

Substituting $\mathcal{C}_{3}$ into Equation (13), we have

$$
\begin{aligned}
\mathcal{C}_{2}=\frac{f_{4}}{K}\left[f_{1}\left(\frac{1}{\Gamma(\beta)} \sum_{i=0}^{\zeta-\beta}(\zeta-\sigma(i))^{(\beta-1)}+\frac{1}{\Gamma(\beta-3)} \sum_{i=0}^{n+3}(\beta+n-\sigma(i))^{(\beta-4)}\right) H(i+\beta-1)\right. \\
\left.-\frac{e_{1}}{\Gamma(\beta-1)} \sum_{i=0}^{n+1}(\beta+n-\sigma(i))^{(\beta-2)} H(i+\beta-1)\right] .
\end{aligned}
$$

By using the value of $\mathcal{C}_{2}$ and $\mathcal{C}_{3}$ in Equation (17), we arrive at 


$$
\begin{aligned}
\mathcal{C}_{1} & =\frac{1}{e_{1} K}\left\{\frac{e_{1} h_{2}}{\Gamma(\beta-1)} \sum_{i=0}^{n+1}(\beta+n-\sigma(i))^{(\beta-2)} H(i+\beta-1)\right. \\
& \left.+h_{1}\left(\frac{1}{\Gamma(\beta)} \sum_{i=0}^{\zeta-\beta}(\zeta-\sigma(i))^{(\beta-1)}+\frac{1}{\Gamma(\beta-3)} \sum_{i=0}^{n+3}(\beta+n-\sigma(i))^{(\beta-4)}\right) H(i+\beta-1)\right\} .
\end{aligned}
$$

By using the constants $\mathcal{C}_{j}$ for $j=1,2,3$ in Equation (9), we obtain $w(\kappa)$ in the form

$$
\begin{aligned}
w(\kappa)= & \frac{1}{\Gamma(\beta)} \sum_{i=0}^{\kappa-\beta}(\kappa-\sigma(i))^{(\beta-1)} H(i+\beta-1) \\
& +\mathbb{E}_{1}(\kappa)\left[\frac{1}{\Gamma(\beta)} \sum_{i=0}^{\zeta-\beta}(\zeta-\sigma(i))^{(\beta-1)}+\frac{1}{\Gamma(\beta-3)} \sum_{i=0}^{n+3}(\beta+n-\sigma(i))^{(\beta-4)}\right] H(i+\beta-1) \\
& +\frac{\mathbb{E}_{2}(\kappa)}{\Gamma(\beta-1)} \sum_{i=0}^{n+1}(\beta+n-\sigma(i))^{(\beta-2)} H(i+\beta-1),
\end{aligned}
$$

for $\kappa \in \mathbb{N}_{\beta-4}^{\beta+n+3}$. Therefore, the theorem's proof is complete.

Assume that $\mathbb{B}_{*}: \mathbb{C}\left(\mathbb{N}_{\beta-4}^{\beta+n+3}, \mathbb{R}\right)$ is a Banach space with a norm defined by

$$
\|w\|=\max \left\{|w(\kappa)|: \kappa \in \mathbb{N}_{\beta-4}^{\beta+n+3}\right\} .
$$

To discuss the theorems' existence and uniqueness, we need the following assumptions:

$\left(A_{1}\right)$ There exists a constant $\mathbb{L}_{G}>0$, which satisfies $|G(\kappa, w)-G(\kappa, \hat{w})| \leq \mathbb{L}_{G}|w-\hat{w}|$ for all $w, \hat{w} \in \mathbb{B}_{*}$ and each $\kappa \in \mathbb{N}_{\beta-4}^{\beta+n+3}$.

$\left(A_{2}\right)$ There exists a bounded function $\chi: \mathbb{N}_{\beta-4}^{\beta+n+3} \rightarrow \mathbb{R}$ with $|G(\kappa, w)| \leq \chi(\kappa)|w|$ for all $w \in \mathbb{B}_{*}$.

Theorem 2. In view of assumption $\left(A_{1}\right)$, the discrete FEBE with the three-point BCs in Equation (2) has a unique solution if

$$
\begin{array}{r}
\Lambda:=\left[\frac{(\beta+n+3)^{(\beta)}}{\Gamma(\beta+1)}+\mathbb{E}_{1}^{*}\left(\frac{\zeta^{(\beta)}}{\Gamma(\beta+1)}+\frac{(\beta+n)^{(\beta-3)}}{\Gamma(\beta-2)}\right)+\mathbb{E}_{2}^{*} \frac{(\beta+n)^{(\beta-1)}}{\Gamma(\beta)}\right] \mathbb{L}_{G}<1, \\
\text { where } \\
\mathbb{E}_{1}^{*}=\left|\frac{1}{K}\left[\frac{(\beta+n+3)^{(\beta-1)}}{e_{1}} h_{1}+(\beta+n+3)^{(\beta-2)} f_{1} f_{4}-(\beta+n+3)^{(\beta-3)} f_{1}\right]\right|, \\
\mathbb{E}_{2}^{*}=\left|\frac{1}{K}\left[(\beta+n+3)^{(\beta-1)} h_{2}-(\beta+n+3)^{(\beta-2)} e_{1} f_{4}+(\beta+n+3)^{(\beta-3)} e_{1}\right]\right|,
\end{array}
$$

such that $K$ is defined in Theorem 1

Proof. Let the operator $\mathcal{A}: \mathbb{B}_{*} \rightarrow \mathbb{B}_{*}$ be defined as

$$
\begin{aligned}
(\mathcal{A} w)(\kappa) & =\frac{1}{\Gamma(\beta)} \sum_{i=0}^{\kappa-\beta}(\kappa-\sigma(i))^{(\beta-1)} g_{w}(\kappa) \\
& +\mathbb{E}_{1}(\kappa)\left[\frac{1}{\Gamma(\beta)} \sum_{i=0}^{\zeta-\beta}(\zeta-\sigma(i))^{(\beta-1)}+\frac{1}{\Gamma(\beta-3)} \sum_{i=0}^{n+3}(\beta+n-\sigma(i))^{(\beta-4)}\right] g_{w}(\kappa) \\
& +\frac{\mathbb{E}_{2}(\kappa)}{\Gamma(\beta-1)} \sum_{i=0}^{n+1}(\beta+n-\sigma(i))^{(\beta-2)} g_{w}(\kappa)
\end{aligned}
$$


where $g_{w}(\kappa)=G(\kappa+\beta-1, w(\kappa+\beta-1))$. Obviously, the fixed point of $\mathcal{A}$ is a solution to Equation (2). To show that $\mathcal{A}$ is a contraction, let $w, \hat{w} \in \mathbb{B}_{*}$ and for each $\kappa \in \mathbb{N}_{\beta-4}^{\beta+n+3}$, one has

$$
\begin{aligned}
|(\mathcal{A} w)(\kappa)-(\mathcal{A} \hat{w})(\kappa)| \leq & \frac{1}{\Gamma(\beta)} \sum_{i=0}^{\kappa-\beta}(\kappa-\sigma(i))^{(\beta-1)}\left|g_{w}(i)-g_{\hat{w}}(i)\right| \\
& +\left|\mathbb{E}_{1}(\kappa)\right|\left[\frac{1}{\Gamma(\beta)} \sum_{i=0}^{\zeta-\beta}(\zeta-\sigma(i))^{(\beta-1)}+\right. \\
& \left.\frac{1}{\Gamma(\beta-3)} \sum_{i=0}^{n+3}(\beta+n-\sigma(i))^{(\beta-4)}\right]\left|g_{w}(i)-g_{\hat{w}}(i)\right| \\
& +\frac{\left|\mathbb{E}_{2}(\kappa)\right|}{\Gamma(\beta-1)} \sum_{i=0}^{n+1}(\beta+n-\sigma(i))^{(\beta-2)}\left|g_{w}(i)-g_{\hat{w}}(i)\right|,
\end{aligned}
$$

where $g_{w}(\kappa), g_{\hat{w}}(\kappa) \in \mathbb{C}\left(\mathbb{N}_{\beta-4}^{\beta+n+3}, \mathbb{R}\right)$ satisfies the following functional equations:

$$
g_{w}(\kappa)=G(\kappa+\beta-1, w(\kappa+\beta-1)) \text { and } g_{\hat{w}}(\kappa)=G(\kappa+\beta-1, \hat{w}(\kappa+\beta-1)) .
$$

By $\left(A_{1}\right)$, we have

$$
\begin{aligned}
\left|g_{w}(\kappa)-g_{\hat{w}}(\kappa)\right| & =|G(\kappa+\beta-1, w(\kappa+\beta-1))-G(\kappa+\beta-1, \hat{w}(\kappa+\beta-1))| \\
& \leq \mathbb{L}_{G}|w(\kappa+\beta-1)-\hat{w}(\kappa+\beta-1)| \\
\left|g_{w}(\kappa)-g_{\hat{w}}(\kappa)\right| & \leq \mathbb{L}_{G}|| w-\hat{w} \| .
\end{aligned}
$$

From which we obtain

$$
\begin{aligned}
\|\mathcal{A} w-\mathcal{A} \hat{w}\| & \leq \frac{\mathbb{L}_{G}\|w-\hat{w}\|}{\Gamma(\beta)} \sum_{i=0}^{\kappa-\beta}(\kappa-\sigma(i))^{(\beta-1)} \\
& +\left|\mathbb{E}_{1}(\kappa)\right| \mathbb{L}_{G}\|w-\hat{w}\|\left[\frac{1}{\Gamma(\beta)} \sum_{i=0}^{\zeta-\beta}(\zeta-\sigma(i))^{(\beta-1)}+\frac{1}{\Gamma(\beta-3)} \sum_{i=0}^{n+3}(\beta+n-\sigma(i))^{(\beta-4)}\right] \\
& +\frac{\left|\mathbb{E}_{2}(\kappa)\right| \mathbb{L}_{G}\|w-\hat{w}\|}{\Gamma(\beta-1)} \sum_{i=0}^{n+1}(\beta+n-\sigma(i))^{(\beta-2)} .
\end{aligned}
$$

By the application of Lemma 3, we have

$$
\frac{1}{\Gamma(\beta)} \sum_{i=0}^{\kappa-\beta}(\kappa-\sigma(i))^{(\beta-1)}=\frac{1}{\Gamma(\beta)}\left[\frac{(\kappa-i)^{(\beta)}}{-\beta}\right]_{i=0}^{\kappa-\beta+1}=\frac{\kappa^{(\beta)}}{\Gamma(\beta+1)} \leq \frac{(\beta+n+3)^{(\beta)}}{\Gamma(\beta+1)}
$$

and

$$
\frac{1}{\Gamma(\beta)} \sum_{i=0}^{\zeta-\beta}(\zeta-\sigma(i))^{(\beta-1)}=\frac{1}{\Gamma(\beta)}\left[\frac{(\zeta-i)^{(\beta)}}{-\beta}\right]_{i=0}^{\zeta-\beta+1}=\frac{\zeta^{(\beta)}}{\Gamma(\beta+1)} .
$$

Similarly, by using Lemma 3, we also obtain

$$
\begin{aligned}
& \frac{1}{\Gamma(\beta-1)} \sum_{i=0}^{n+1}(\beta+n-\sigma(i))^{(\beta-2)}=\frac{1}{\Gamma(\beta-1)}\left[\frac{(\beta+n-i)^{(\beta-1)}}{-(\beta-1)}\right]_{i=0}^{n+2}=\frac{(\beta+n)^{(\beta-1)}}{\Gamma(\beta)} \\
& \text { and } \\
& \frac{1}{\Gamma(\beta-3)} \sum_{i=0}^{n+3}(\beta+n-\sigma(i))^{(\beta-4)}=\frac{1}{\Gamma(\beta-3)}\left[\frac{(\beta+n-i)^{(\beta-3)}}{-(\beta-3)}\right]_{i=0}^{n+4}=\frac{(\beta+n)^{(\beta-3)}}{\Gamma(\beta-2)} .
\end{aligned}
$$


By substituting the relations Equations (25)-(28) into Equation (24), we obtain

$$
\|\mathcal{A} w-\mathcal{A} w\| \leq\left[\frac{(\beta+n+3)^{(\beta)}}{\Gamma(\beta+1)}+\mathbb{E}_{1}^{*}\left(\frac{\zeta^{(\beta)}}{\Gamma(\beta+1)}+\frac{(\beta+n)^{(\beta-3)}}{\Gamma(\beta-2)}\right)+\mathbb{E}_{2}^{*} \frac{(\beta+n)^{(\beta-1)}}{\Gamma(\beta)}\right] \mathbb{L}_{G}\|w-\hat{w}\| .
$$

By Equation (19), we obtain $\|\mathcal{A} w-\mathcal{A} \hat{w}\|<\|w-\hat{w}\|$. Hence, $\mathcal{A}$ is a contraction. As a result, according to the Banach fixed-point theorem, the three-point $B C s$ for the discrete FEBE Equation (2) has a unique solution.

Theorem 3. If the assumption $\left(A_{2}\right)$ holds, then the discrete FEBE with three-point BCs in Equation (2) has at least one solution provided that

$$
\chi^{*} \leq \frac{\Gamma(\beta+1)}{\left[(\beta+n+3)^{(\beta)}+\mathbb{E}_{1}^{*}\left(\zeta^{(\beta)}+\beta^{(3)}(\beta+n)^{(\beta-3)}\right)+\mathbb{E}_{2}^{*} \beta(\beta+n)^{(\beta-1)}\right]},
$$

where $\chi^{*}=\max \left\{\chi(\kappa): \kappa \in \mathbb{N}_{\beta-4}^{\beta+n+3}\right\}$.

Proof. Assume that $D>0$ and consider the set $V=\left\{w \in \mathbb{B}_{*}:\|w\| \leq D\right\}$. For proving this theorem, let us claim that $\mathcal{A}$ maps $V$ in $V$. Now, for any $w \in V$, one has

$$
\begin{aligned}
|(\mathcal{A} w)(\kappa)| & \leq \frac{1}{\Gamma(\beta)} \sum_{i=0}^{\kappa-\beta}(\kappa-\sigma(i))^{(\beta-1)}\left|g_{w}(i)\right| \\
& +\left|\mathbb{E}_{1}(\kappa)\right|\left[\frac{1}{\Gamma(\beta)} \sum_{i=0}^{\zeta-\beta}(\zeta-\sigma(i))^{(\beta-1)}+\frac{1}{\Gamma(\beta-3)} \sum_{i=0}^{n+3}(\beta+n-\sigma(i))^{(\beta-4)}\right]\left|g_{w}(i)\right| \\
& +\frac{\left|\mathbb{E}_{2}(\kappa)\right|}{\Gamma(\beta-1)} \sum_{i=0}^{n+1}(\beta+n-\sigma(i))^{(\beta-2)}\left|g_{w}(i)\right|,
\end{aligned}
$$

where $g_{w}(\kappa)$ is given in Equation (22). Using $\left(A_{2}\right)$, we obtain

$$
\left|g_{w}(\kappa)\right|=|G(\kappa+\beta-1, w(\kappa+\beta-1))| \leq \chi(\kappa)|w(\kappa+\beta-1)| \leq \chi^{*}\|w\| .
$$

This further implies that

$$
\begin{aligned}
\|\mathcal{A} w\| & \leq \frac{\chi^{*}\|w\|}{\Gamma(\beta)} \sum_{i=0}^{\kappa-\beta}(\kappa-\sigma(i))^{(\beta-1)} \\
& +\left|\mathbb{E}_{1}(\kappa)\right| \chi^{*}\|w\|\left[\frac{1}{\Gamma(\beta)} \sum_{i=0}^{\zeta-\beta}(\zeta-\sigma(i))^{(\beta-1)}+\frac{1}{\Gamma(\beta-3)} \sum_{i=0}^{n+3}(\beta+n-\sigma(i))^{(\beta-4)}\right] \\
& +\frac{\left|\mathbb{E}_{2}(\kappa)\right| \chi^{*}\|w\|}{\Gamma(\beta-1)} \sum_{i=0}^{n+1}(\beta+n-\sigma(i))^{(\beta-2)} .
\end{aligned}
$$

Using the relations of Equations (25)-(28) in Equation (30), we obtain

$$
\|\mathcal{A} w\| \leq\left[\frac{(\beta+n+3)^{(\beta)}+\mathbb{E}_{1}^{*}\left(\zeta^{(\beta)}+\beta^{(3)}(\beta+n)^{(\beta-3)}\right)+\mathbb{E}_{2}^{*} \beta(\beta+n)^{(\beta-1)}}{\Gamma(\beta+1)}\right] \chi^{*} D .
$$

By Equation (29), we have $\|\mathcal{A} w\| \leq D$, which implies that $\mathcal{A}: V \rightarrow V$. By using the Brouwer fixed-point theorem, let us conclude that three-point BCs for discrete FEBE Equation (2) has at least one solution. 


\section{EB Stability Analysis}

The Ulam-type stability for the proposed problem Equation (2) is studied in this section. Now, we present some definitions of Ulam stability, and we also assume that $g_{\hat{w}}(\kappa)$ : $\mathbb{C}\left(\mathbb{N}_{\beta-4}^{\beta+n+3}, \mathbb{R}\right)$ is a continuous function that satisfies $g_{\hat{w}}(\kappa)=G(\kappa+\beta-1, \hat{w}(\kappa+\beta-1))$.

Definition 2 ([46]). If for every function $\hat{w} \in \mathbb{B}_{*}$ of

$$
\left|\Delta_{\beta-4}^{\beta} \hat{w}(\kappa)-g_{\hat{w}}(\kappa)\right| \leq \epsilon, \quad \kappa \in \mathbb{N}_{0}^{n+3},
$$

where $\epsilon>0$, there exists solution $w \in \mathbb{B}_{*}$ of Equation (2) and positive number $\delta_{1}>0$ such that

$$
|\hat{w}(\kappa)-w(\kappa)| \leq \delta_{1} \epsilon, \quad \kappa \in \mathbb{N}_{\beta-4}^{\beta+n+3} .
$$

Then, the discrete FEBE Equation (2) is $\mathcal{H U}$ stable. It will be $\mathcal{G H U}$ stable if we keep $\Phi(\epsilon)=$ $\delta_{1} \epsilon$ in inequality Equation (32), where $\Phi(\epsilon) \in \mathbb{C}\left(\mathbb{R}^{+}, \mathbb{R}^{+}\right)$and $\Phi(0)=0$.

Definition 3 ([46]). If for every function $\hat{w} \in \mathbb{B}_{*}$ of

$$
\left|\Delta_{\beta-4}^{\beta} \hat{w}(\kappa)-g_{\hat{w}}(\kappa)\right| \leq \epsilon \phi(\kappa+\beta-1), \quad \kappa \in \mathbb{N}_{0}^{n+3},
$$

where $\epsilon>0$, there are solutions $w \in \mathbb{B}_{*}$ of Equation (2) and positive number $\delta_{2}>0$ such that

$$
|\hat{w}(\kappa)-w(\kappa)| \leq \delta_{2} \epsilon \phi(\kappa+\beta-1), \kappa \in \mathbb{N}_{\beta-4}^{\beta+n+3} .
$$

Then, the discrete FEBE Equation (2) is $\mathcal{H U R}$ stable. It will be $\mathcal{G H U R}$ stable if $\phi(\kappa+\beta-1)=\epsilon \phi(\kappa+\beta-1)$ in inequality Equations (33) and (34).

Remark 1 ([46]). A function $\hat{w} \in \mathbb{B}_{*}$ is a solution to Equation (31) iff there exists $\Psi: \mathbb{N}_{\beta-4}^{\beta+n+3} \rightarrow$ $\mathbb{R}$ that satisifies, for $\kappa \in \mathbb{N}_{0}^{n+3}$, the following:

$\left(A_{3}\right)|\Psi(\kappa+\beta-1)| \leq \epsilon$,

$\left(A_{4}\right) \Delta_{\beta-4}^{\beta} \hat{w}(\kappa)=g_{\hat{w}}(\kappa)+\Psi(\kappa+\beta-1)$.

Similarly, a remark can be constructed for inequality Equation (33).

Lemma 5. According to Remark 1, a function $\hat{w} \in \mathbb{B}_{*}$ that corresponds to the discrete FEBE with three-point $B C$ s is expressed as:

$$
\left\{\begin{array}{l}
\Delta_{\beta-4}^{\beta} \hat{w}(\kappa)=g_{\hat{w}}(\kappa)+\Psi(\kappa+\beta-1), \kappa \in \mathbb{N}_{0}^{n+3}, \\
w(\beta-4)=0, \Delta^{2} w(\beta-4)=0, \Delta w(\beta+n)=0, \Delta^{3} w(\beta+n)+w(\zeta)=0,
\end{array}\right.
$$

satisfying the following inequality:

$$
|\hat{w}(\kappa)-(\mathcal{A} \hat{w})(\kappa)| \leq \frac{\epsilon}{\Gamma(\beta+1)}(\beta+n+3)^{(\beta)},
$$

where $(\mathcal{A} \hat{w})(\kappa)$ is defined in Equation (21). 
Proof. By using Theorem 1, the corresponding BVP Equation (35) becomes

$$
\begin{aligned}
\hat{w}(\kappa)= & \frac{1}{\Gamma(\beta)} \sum_{i=0}^{\kappa-\beta}(\kappa-\sigma(i))^{(\beta-1)} g_{\hat{w}}(i) \\
& +\mathbb{E}_{1}(\kappa)\left[\frac{1}{\Gamma(\beta)} \sum_{i=0}^{\zeta-\beta}(\zeta-\sigma(i))^{(\beta-1)}+\frac{1}{\Gamma(\beta-3)} \sum_{i=0}^{n+3}(\beta+n-\sigma(i))^{(\beta-4)}\right] g_{\hat{w}}(i) \\
& +\frac{\mathbb{E}_{2}(\kappa)}{\Gamma(\beta-1)} \sum_{i=0}^{n+1}(\beta+n-\sigma(i))^{(\beta-2)} g_{\hat{w}}(i) \\
& +\frac{1}{\Gamma(\beta)} \sum_{i=0}^{\kappa-\beta}(\kappa-\sigma(i))^{(\beta-1)} \Psi(i+\beta-1) .
\end{aligned}
$$

Using an operator $\mathcal{A}$ and taking the modulus on both sides of the above solution along with $\left(A_{3}\right)$, we obtain

$$
|\hat{w}(\kappa)-(\mathcal{A} \hat{w})(\kappa)| \leq \frac{\epsilon}{\Gamma(\beta+1)}(\beta+n+3)^{(\beta)} .
$$

Theorem 4. Under the assumption $\left(A_{1}\right)$ with the inequality Equation (19), the discrete FEBE Equation (2) is $\mathrm{HU}$ stable.

Proof. If $\hat{w}(\kappa)$ is any solution of the inequality Equation (31), and $w(\kappa)$ is a unique solution to Equation (2), then

$$
\begin{aligned}
|\hat{w}(\kappa)-w(\kappa)| & =|\hat{w}(\kappa)-(\mathcal{A} w)(\kappa)| \\
& =|\hat{w}(\kappa)-(\mathcal{A} \hat{w})(\kappa)+(\mathcal{A} \hat{w})(\kappa)-(\mathcal{A} w)(\kappa)| \\
& \leq|\hat{w}(\kappa)-(\mathcal{A} \hat{w})(\kappa)|+|(\mathcal{A} \hat{w})(\kappa)-(\mathcal{A} w)(\kappa)| .
\end{aligned}
$$

By using Lemma 5 in Equation (36), we have

$$
\begin{aligned}
|\hat{w}(\kappa)-w(\kappa)| \leq & \frac{\epsilon}{\Gamma(\beta+1)}(\beta+n+3)^{(\beta)} \\
& +\left[\frac{(\beta+n+3)^{(\beta)}}{\Gamma(\beta+1)}+\mathbb{E}_{1}^{*}\left(\frac{\zeta^{(\beta)}}{\Gamma(\beta+1)}+\frac{(\beta+n)^{(\beta-3)}}{\Gamma(\beta-2)}\right)+\mathbb{E}_{2}^{*} \frac{(\beta+n)^{(\beta-1)}}{\Gamma(\beta)}\right] \mathbb{L}_{G}\|\hat{w}-w\| .
\end{aligned}
$$

This further implies that

$$
\|\hat{w}-w\| \leq \delta_{1} \epsilon
$$

where

$$
\delta_{1}=\frac{(\beta+n+3)^{(\beta)}}{\Gamma(\beta+1)-\mathbb{L}_{G}\left[(\beta+n+3)^{(\beta)}+\mathbb{E}_{1}^{*}\left(\zeta^{(\beta)}+\beta^{(3)}(\beta+n)^{(\beta-3)}\right)+\mathbb{E}_{2}^{*} \beta(\beta+n)^{(\beta-1)}\right]} .
$$

Hence, the solution of Equation (2) is $\mathcal{H U}$ stable.

Remark 2. If $\Phi(\epsilon)=\delta_{1} \epsilon$ such that $\Phi(0)=0$, then we have

$$
\|\hat{w}-w\| \leq \Phi(\epsilon) .
$$

Hence, the solution of Equation (2) is $\mathcal{G H U}$ stable.

For our next result, the following hypotheses hold: 
( $A_{5}$ ) For an increasing function $\phi \in \mathbb{N}_{\beta-4}^{\beta+n+3} \rightarrow \mathbb{R}^{+}$, there exists $\lambda>0$ such that, for $\kappa \in \mathbb{N}_{0}^{n+3}$
(i) $\frac{\epsilon}{\Gamma(\beta)} \sum_{i=0}^{\kappa-\beta}(\kappa-\sigma(i))^{(\beta-1)} \phi(i+\beta-1) \leq \lambda \epsilon \phi(\kappa+\beta-1)$,
(ii) $\frac{1}{\Gamma(\beta)} \sum_{i=0}^{\kappa-\beta}(\kappa-\sigma(i))^{(\beta-1)} \phi(i+\beta-1) \leq \lambda \phi(\kappa+\beta-1)$.

Lemma 6. For the three-point BCs of discrete FEBE Equation (35), the following inequality holds:

$$
|\hat{w}(\kappa)-(\mathcal{A} \hat{w})(\kappa)| \leq \lambda \epsilon \phi(\kappa+\beta-1),
$$

where $(\mathcal{A} \hat{w})(\kappa)$ is defined in Equation (21).

Proof. From inequality Equation (33), for $\kappa \in \mathbb{N}_{\beta-4}^{\beta+n+3}$, we obtain a function $\Delta_{\beta-4}^{\beta} \hat{w}(\kappa)=$ $g_{\hat{w}}(\kappa)+\Psi(\kappa+\beta-1),|\Psi(\kappa+\beta-1)| \leq \epsilon \phi(\kappa+\beta-1)$ and $\left(A_{5}\right)(\mathrm{i})$ such that

$$
|\hat{w}(\kappa)-(\mathcal{A} \hat{w})(\kappa)| \leq \lambda \epsilon \phi(\kappa+\beta-1) .
$$

Theorem 5. Under the hypothesis $\left(A_{1}\right)$ with the inequality Equation (19), the discrete FEBE Equation (2) is $\mathcal{H} \mathcal{U} \mathcal{R}$ stable.

Proof. By using a similar procedure of Theorem 4 together with Lemma 6 for $\kappa \in \mathbb{N}_{\beta-4}^{\beta+n+3}$, we obtain

$$
\begin{aligned}
|\hat{w}(\kappa)-w(\kappa)| \leq & \lambda \epsilon \phi(\kappa+\beta-1) \\
& +\left[\frac{(\beta+n+3)^{(\beta)}}{\Gamma(\beta+1)}+\mathbb{E}_{1}^{*}\left(\frac{\zeta^{(\beta)}}{\Gamma(\beta+1)}+\frac{(\beta+n)^{(\beta-3)}}{\Gamma(\beta-2)}\right)+\mathbb{E}_{2}^{*} \frac{(\beta+n)^{(\beta-1)}}{\Gamma(\beta)}\right] \mathbb{L}_{G}\|\hat{w}-w\| .
\end{aligned}
$$

This further implies that

$$
\|\hat{w}-w\| \leq \delta_{2} \epsilon \phi(\kappa+\beta-1)
$$

where

$\delta_{2}=\frac{\lambda \Gamma(\beta+1)}{\Gamma(\beta+1)-\mathbb{L}_{G}\left[(\beta+n+3)^{(\beta)}+\mathbb{E}_{1}^{*}\left(\zeta^{(\beta)}+\beta^{(3)}(\beta+n)^{(\beta-3)}\right)+\mathbb{E}_{2}^{*} \beta(\beta+n)^{(\beta-1)}\right]}$.

Thus, the solution of Equation (2) is $\mathcal{H} \mathcal{U} \mathcal{R}$ stable.

Remark 3. If $\phi(\kappa+\beta-1)=\epsilon \phi(\kappa+\beta-1)$, then we have

$$
\|\hat{w}-w\| \leq \delta_{2} \phi(\kappa+\beta-1) .
$$

Hence, the solution of Equation (2) is $\mathcal{G H U \mathcal { R }}$ stable.

\section{Applications}

Some illustrative examples are provided in this section to demonstrate the applicability of our results in this research work.

Example 1. Suppose that $\beta=3.7, n=2$, and $H(\kappa)=\kappa^{(13)}$ with different values of $\zeta$. Then, $a$ linear discrete FEBE with the three-point BCs of Equation (3) becomes

$$
\left\{\begin{array}{l}
\Delta_{-0.3}^{3.7} w(\kappa)=(\kappa+2.7)^{(13)}, \kappa \in \mathbb{N}_{0}^{5}, \\
w(-0.3)=0, \Delta^{2} w(-0.3)=0, \Delta w(5.7)=0, \Delta^{3} w(5.7)+w(\zeta)=0 .
\end{array}\right.
$$


We shall apply Theorem 1 to find a solution $w(\kappa)$ of Equation (37) that can be expressed as:

$$
\begin{aligned}
w(\kappa)= & \frac{1}{\Gamma(3.7)} \sum_{i=0}^{\kappa-3.7}(\kappa-\sigma(i))^{(2.7)}(i+2.7)^{(13)} \\
& +\mathbb{E}_{1}(\kappa)\left[\frac{1}{\Gamma(3.7)} \sum_{i=0}^{\zeta-3.7}(\zeta-\sigma(i))^{(2.7)}+\frac{1}{\Gamma(0.7)} \sum_{i=0}^{5}(5.7-\sigma(i))^{(-0.3)}\right](i+2.7)^{(13)} \\
& +\frac{\mathbb{E}_{2}(\kappa)}{\Gamma(2.7)} \sum_{i=0}^{3}(5.7-\sigma(i))^{(1.7)}(i+2.7)^{(13)},
\end{aligned}
$$

where $\kappa \in \mathbb{N}_{-0.3}^{8.7}, \mathbb{E}_{1}(\kappa)$ and $\mathbb{E}_{2}(\kappa)$ are defined in Theorem 1. With the help of both Definition 1 and Lemma 4, we obtain the expression on right-hand side of Equation (38) as follows:

$$
\begin{aligned}
\frac{1}{\Gamma(3.7)} \sum_{i=0}^{\kappa-3.7}(\kappa-\sigma(i))^{(2.7)}(i+2.7)^{(13)} & =\Delta^{-3.7}(\kappa+2.7)^{(13)} \\
& =\frac{\Gamma(14)}{\Gamma(17.7)} \cdot \frac{\Gamma(\kappa+3.7)}{\Gamma(\kappa-13)}
\end{aligned}
$$

Similarly, we find

$$
\begin{gathered}
\frac{1}{\Gamma(3.7)} \sum_{i=0}^{\zeta-3.7}(\zeta-\sigma(i))^{(2.7)}(i+2.7)^{(13)}=\frac{\Gamma(14)}{\Gamma(17.7)} \cdot \frac{\Gamma(\zeta+3.7)}{\Gamma(\zeta-13)} \\
\frac{1}{\Gamma(2.7)} \sum_{i=0}^{3}(5.7-\sigma(i))^{(1.7)}(i+2.7)^{(13)}=\frac{\Gamma(14)}{\Gamma(16.7)} \cdot \frac{\Gamma(9.4)}{\Gamma(-6.3)} \\
\frac{1}{\Gamma(0.7)} \sum_{i=0}^{5}(5.7-\sigma(i))^{(-0.3)}(i+2.7)^{(13)}=\frac{\Gamma(14)}{\Gamma(14.7)} \cdot \frac{\Gamma(9.4)}{\Gamma(-4.3)}
\end{gathered}
$$

By substituting the expressions Equations (39)-(42) into Equation (38), we obtain Equation (37)'s solution for $\kappa \in \mathbb{N}_{-0.3}^{8.7}$, in the form

$$
\begin{aligned}
w(\kappa)= & {\left[\frac{\Gamma(14)}{\Gamma(17.7)} \cdot \frac{\Gamma(\kappa+3.7)}{\Gamma(\kappa-13)}\right]+\mathbb{E}_{2}(\kappa)\left[\frac{\Gamma(14)}{\Gamma(16.7)} \cdot \frac{\Gamma(9.4)}{\Gamma(-6.3)}\right] } \\
& +\mathbb{E}_{1}(\kappa)\left[\left(\frac{\Gamma(14)}{\Gamma(17.7)} \cdot \frac{\Gamma(\zeta+3.7)}{\Gamma(\zeta-13)}\right)+\left(\frac{\Gamma(14)}{\Gamma(14.7)} \cdot \frac{\Gamma(9.4)}{\Gamma(-4.3)}\right)\right] .
\end{aligned}
$$

On one hand, by choosing different values of $\zeta=2.7,3.7,4.7,5.7$ in Equation (43), we obtain different solutions for this problem, as seen in Figure 1a. On the other hand, Figure $1 b$ shows three-dimensional solution surface plots for various values $\kappa$ and $\zeta$. In addition, a numerical experiment for our obtained solutions in Example 1 with step size 1 is presented in Table 1. 


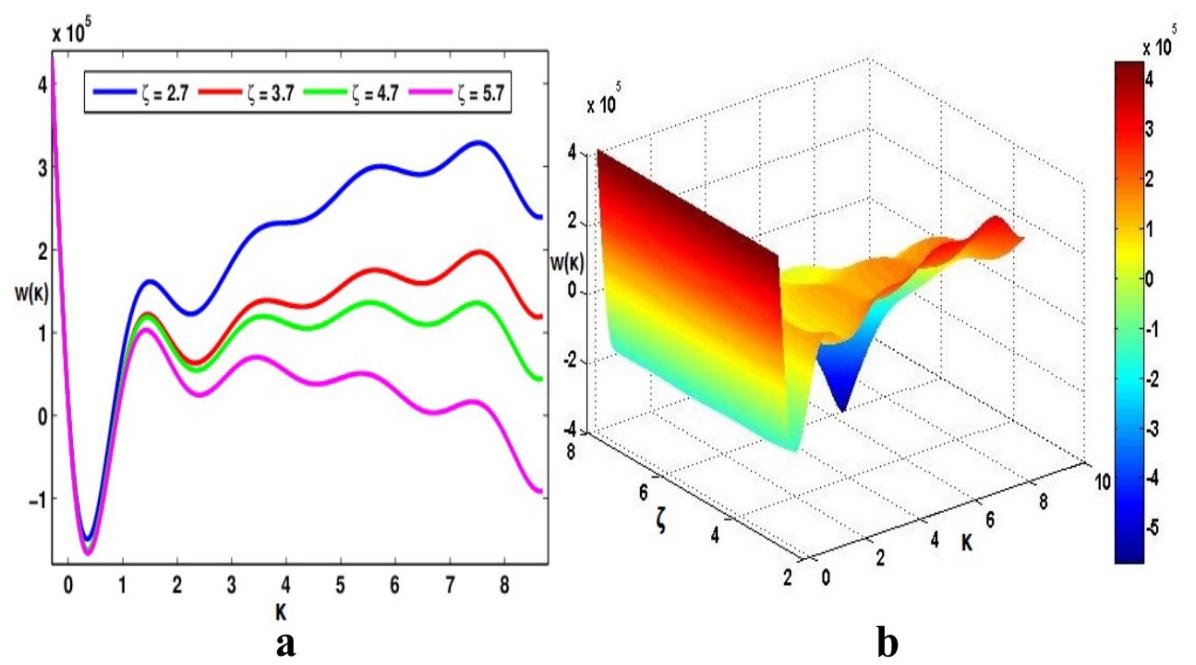

Figure 1. (a) Solution curves for various values of $\zeta$ of a discrete FEBE with the three-point BCs of Equation (37); (b) surface plots for different values of $\kappa$ and $\zeta$ corresponding to Figure 1a.

Table 1. Numerical values of $w(\kappa)$ for Example 1 with step size 1.

\begin{tabular}{ccccc}
\hline \multicolumn{5}{c}{$\boldsymbol{w}(\boldsymbol{\kappa})$} \\
\hline $\boldsymbol{\zeta} \zeta$ & 2.7 & 3.7 & 4.7 & 5.7 \\
\hline-0.3 & $4.3158 \times 10^{5}$ & $4.3158 \times 10^{5}$ & $4.3158 \times 10^{5}$ & $4.3158 \times 10^{5}$ \\
0.7 & $-0.5233 \times 10^{5}$ & $-0.7356 \times 10^{5}$ & $-0.7410 \times 10^{5}$ & $-0.8078 \times 10^{5}$ \\
1.7 & $1.5354 \times 10^{5}$ & $1.0872 \times 10^{5}$ & $1.0384 \times 10^{5}$ & $0.8468 \times 10^{5}$ \\
2.7 & $1.4988 \times 10^{5}$ & $0.8076 \times 10^{5}$ & $0.6918 \times 10^{5}$ & $0.3413 \times 10^{5}$ \\
3.7 & $2.3044 \times 10^{5}$ & $1.3849 \times 10^{5}$ & $1.1846 \times 10^{5}$ & $0.6559 \times 10^{5}$ \\
4.7 & $2.5123 \times 10^{5}$ & $1.3991 \times 10^{5}$ & $1.1014 \times 10^{5}$ & $0.3865 \times 10^{5}$ \\
5.7 & $3.0038 \times 10^{5}$ & $1.7498 \times 10^{5}$ & $1.3450 \times 10^{5}$ & $0.4457 \times 10^{5}$ \\
6.7 & $2.9459 \times 10^{5}$ & $1.6211 \times 10^{5}$ & $1.1023 \times 10^{5}$ & $0.0290 \times 10^{5}$ \\
7.7 & $3.2506 \times 10^{5}$ & $1.9406 \times 10^{5}$ & $1.3037 \times 10^{5}$ & $0.0745 \times 10^{5}$ \\
8.7 & $2.3972 \times 10^{5}$ & $1.2029 \times 10^{5}$ & $0.4457 \times 10^{5}$ & $-0.9137 \times 10^{5}$ \\
\hline
\end{tabular}

Example 2. Consider a discrete FEBE subject to three-point BCs:

$$
\begin{aligned}
& \left\{\begin{array}{l}
\Delta_{\pi-4}^{\pi} w(\kappa)=\frac{1}{(\kappa+\pi-1)+650}\left[\sin (w(\kappa+\pi-1))+\frac{e^{-(\kappa+\pi-1)} \cos (\kappa+\pi-1)}{10 \sqrt{\pi}(\kappa+\pi)}\right], \kappa \in \mathbb{N}_{0}^{6}, \\
w(\pi-4)=0, \Delta^{2} w(\pi-4)=0, \Delta w(\pi+3)=0, \Delta^{3} w(\pi+3)+w(2.1416)=0 .
\end{array}\right. \\
& \text { Clearly, } \beta=\pi, n=3, \zeta=2.1416 . \text { Set } G(\kappa, w(\kappa))=\frac{1}{\kappa+650}\left[\sin (w(\kappa))+\frac{e^{-t} \cos (\kappa)}{10 \sqrt{\pi}(1+\kappa)}\right] \\
& |G(\kappa, w(\kappa))-G(\kappa, \hat{w}(\kappa))|=\frac{1}{\kappa+650}\left|\sin (w(\kappa))+\frac{e^{-t} \cos (\kappa)}{10 \sqrt{\pi}(1+\kappa)}-\sin (\hat{w}(\kappa))-\frac{e^{-t} \cos (\kappa)}{10 \sqrt{\pi}(1+\kappa)}\right| \\
& =\frac{1}{\kappa+650}|\sin (w(\kappa))-\sin (\hat{w}(\kappa))| \\
& |G(\kappa, w(\kappa))-G(\kappa, \hat{w}(\kappa))| \leq 0.0015|w(\kappa)-\hat{w}(\kappa)| .
\end{aligned}
$$

So, we have $\mathbb{L}_{G}=0.0015$, and $G$ is Lipschitz continuous for for $\kappa \in \mathbb{N}_{\pi-4}^{\pi+6}$. Furthermore, the inequality Equation (19) is satisfied with $\Lambda \approx 0.2944<1$. Therefore, from Theorem 2, we conclude that problem Equation (44) has a unique solution. 
Example 3. Assume that $\beta=3.6, n=4$, and $\zeta=2.6$ with $G(\kappa, w(\kappa))=\frac{\kappa}{100} e^{-\frac{w^{2}(\kappa)}{100}}$. Then, we obtain the following discrete FEBE Equation (2) with BCs:

$$
\left\{\begin{array}{l}
\Delta_{-0.4}^{3.6} w(\kappa)=\frac{1}{100}(\kappa+2.6) e^{-\frac{1}{100} w^{2}(\kappa+2.6)}, \kappa \in \mathbb{N}_{0}^{7} \\
w(-0.4)=0, \Delta^{2} w(-0.4)=0, \Delta w(7.6)=0, \Delta^{3} w(7.6)+w(2.6)=0 .
\end{array}\right.
$$

Let a Banach space be $\mathbb{B}_{*}:=\left\{w(\kappa) \mid \mathbb{N}_{-0.4}^{10.6} \rightarrow \mathbb{R}\right\}$. Suppose that $D=1000$. To verify that the hypotheses of Theorem 3 hold, it is noticeable that

$$
\frac{D \Gamma(\beta+1)}{\left[(\beta+n+3)^{(\beta)}+\mathbb{E}_{1}^{*}\left(\zeta^{(\beta)}+\beta^{(3)}(\beta+n)^{(\beta-3)}\right)+\mathbb{E}_{2}^{*} \beta(\beta+n)^{(\beta-1)}\right]} \approx 2.1790 .
$$

Clearly, we have $|G(\kappa, w(\kappa))|=0.1060 \leq 2.1790$, whenever $\|w\| \leq 1000$. Thus, the problem Equation (45) has at least one solution.

Example 4. Consider the discrete FEBE with three-point BCS as follows:

$$
\left\{\begin{array}{l}
\Delta_{-0.8}^{3.2} w(\kappa)=\frac{1}{700} \cos (w(\kappa+2.2))+\frac{1}{((\kappa+2.2)+950)}(\kappa+2.2)^{(3.2)}, \kappa \in \mathbb{N}_{0}^{5} \\
w(-0.8)=0, \Delta^{2} w(-0.8)=0, \Delta w(5.2)=0, \Delta^{3} w(5.2)+w(4.2)=0 .
\end{array}\right.
$$

Here, we have $\beta=3.2, n=2, \zeta=4.2$ and $G(\kappa, w(\kappa))=\frac{1}{700} \cos (w(\kappa))+\frac{1}{(\kappa+950)} \kappa^{(3.2)}$ for $\kappa \in \mathbb{N}_{-0.8}^{8.2}$. Now, we prove that Equation (46) is $\mathcal{H U}$ stable. Since $\left(A_{1}\right)$ holds for each $\kappa \in \mathbb{N}_{-0.8^{\prime}}^{8.2}$ we obtain

$$
\begin{aligned}
|G(\kappa, \hat{w}(\kappa))-G(\kappa, w(\kappa))| & =\left|\frac{1}{700} \cos (\hat{w}(\kappa))+\frac{1}{(\kappa+950)} \kappa^{(3.2)}-\frac{1}{700} \cos (w(\kappa))-\frac{1}{(\kappa+950)} \kappa^{(3.2)}\right| \\
& =\frac{1}{700}|\cos (\hat{w}(\kappa))-\cos (w(\kappa))| \\
|G(\kappa, \hat{w}(\kappa))-G(\kappa, w(\kappa))| & \leq 0.0014|\hat{w}(\kappa)-w(\kappa)|
\end{aligned}
$$$$
\text { so } \mathbb{L}_{G}=0.0014 \text { and } G \text { is Lipschitz continuous for } \kappa \in \mathbb{N}_{-0.8}^{8.2} \text {. Since }
$$

$$
\frac{1}{\left[\frac{(\beta+n+3)^{(\beta)}}{\Gamma(\beta+1)}+\mathbb{E}_{1}^{*}\left(\frac{\zeta^{(\beta)}}{\Gamma(\beta+1)}+\frac{(\beta+n)^{(\beta-3)}}{\Gamma(\beta-2)}\right)+\mathbb{E}_{2}^{*} \frac{(\beta+n)^{(\beta-1)}}{\Gamma(\beta)}\right]} \approx 0.0080,
$$

if $\mathbb{L}_{G}=0.0014<0.0080$. Furthermore, to verify the stability results, from Theorem 4 , we see that $\Lambda=0.1758<1$. Hence, the solution of Equation (46) is $\mathcal{H U}$ stable with $\delta_{1}=80.8287$. In addition, it is $\mathcal{G H U}$ stable from Remark 2. For illustration, we take $\epsilon=0.6017$ and $\hat{w}(\kappa)=\frac{\kappa^{(4)}}{350}$. We prove that Equation (31) holds. Indeed,

$$
\begin{aligned}
\mid \Delta_{-0.8}^{3.2} \hat{w}(\kappa)- & G(\kappa+2.2, \hat{w}(\kappa+2.2)) \mid \\
= & \left|\Delta_{-0.8}^{3.2} \hat{w}(\kappa)-\frac{\cos (\hat{w}(\kappa+2.2))}{700}-\frac{(\kappa+2.2)^{(3.2)}}{\kappa+952.2}\right| \\
= & \left|\Delta_{-0.8}^{3.2}\left(\frac{\kappa^{(4)}}{350}\right)-0.0014 \cos \left[\frac{(\kappa+2.2)^{(4)}}{350}\right]-\frac{(\kappa+2.2)^{(3.2)}}{\kappa+952.2}\right|
\end{aligned}
$$


By using Lemma 4, Equation (47) becomes

$$
\begin{aligned}
\mid \Delta_{-0.8}^{3.2} \hat{w}(\kappa)- & G(\kappa+2.2, \hat{w}(\kappa+2.2)) \mid \\
& =\left|0.0736 \kappa^{(0.8)}-0.0014 \cos \left[\frac{\Gamma(\kappa+3.2)}{350 \Gamma(\kappa-0.8)}\right]-\frac{\Gamma(\kappa+3.2)}{(\kappa+952.2) \Gamma(\kappa)}\right| \\
\leq & 0.0736\left[\frac{\Gamma(\kappa+1)}{\Gamma(\kappa+0.2)}\right]+0.0014+\frac{1}{(\kappa+952.2)}\left[\frac{\Gamma(\kappa+3.2)}{\Gamma(\kappa)}\right] \\
\leq & 0.6017 \leq \epsilon, \text { for } \kappa \in \mathbb{N}_{0}^{5} .
\end{aligned}
$$

Example 5. Consider a discrete FEBE subject to the three-point BCs:

$$
\left\{\begin{array}{l}
\Delta_{\pi-4}^{\pi} w(\kappa)=\frac{1}{700} \sin (w(\kappa+\pi-1))+\frac{1}{310}(\kappa+\pi-1)^{(\pi)}, \kappa \in \mathbb{N}_{0}^{4} \\
w(\pi-4)=0, \Delta^{2} w(\pi-4)=0, \Delta w(\pi+1)=0, \Delta^{3} w(\pi+1)+w(2.1416)=0 .
\end{array}\right.
$$

In this example, $\beta=\pi, n=1, \zeta=2.1416$. Set $G(\kappa, w(\kappa))=\frac{1}{700} \sin (w(\kappa))+\frac{1}{310} \kappa^{(\pi)}$ for $\kappa \in \mathbb{N}_{\pi-4}^{\pi+4}$. Now, we show that Equation (48) is $\mathcal{H U} \mathcal{R}$ stable. For any $\hat{w}, w \in \mathbb{B}_{*}$ and each $\kappa \in \mathbb{N}_{\pi-4}^{\pi+4}$, we obtain

$$
\begin{aligned}
|G(\kappa, \hat{w}(\kappa))-G(\kappa, w(\kappa))| & =\left|\frac{1}{700} \sin (\hat{w}(\kappa))+\frac{1}{310} \kappa^{(\pi)}-\frac{1}{700} \sin (w(\kappa))-\frac{1}{310} \kappa^{(\pi)}\right| \\
& =\frac{1}{700}|\sin (\hat{w}(\kappa))-\sin (w(\kappa))| \\
|G(\kappa, \hat{w}(\kappa))-G(\kappa, w(\kappa))| & \leq 0.0014|\hat{w}(\kappa)-w(\kappa)| .
\end{aligned}
$$

This satisfies $\left(A_{1}\right)$ with $\mathbb{L}_{G}=0.0014$, and $G$ is Lipschitz continuous for $\kappa \in \mathbb{N}_{\pi-4}^{\pi+4}$. Further, by assuming $\epsilon=0.6519$ and $\phi(\kappa+\pi-1)=1$, we have

$$
\begin{aligned}
\frac{0.6519}{\Gamma(\pi)} \sum_{i=0}^{\kappa-\pi}(\kappa-\sigma(i))^{(\pi-1)}(1) & =\frac{(0.6519) \Gamma(\kappa+1)}{\Gamma(\pi+1) \Gamma(\kappa+1-\pi)} \\
& \leq \frac{(0.6519) \Gamma(5)}{\Gamma(\pi+1) \Gamma(5-\pi)} \\
\frac{0.6519}{\Gamma(\pi)} \sum_{i=0}^{\kappa-\pi}(\kappa-\sigma(i))^{(\pi-1)}(1) & \leq 2.2955, \kappa \in \mathbb{N}_{0}^{4} .
\end{aligned}
$$

Thus, $\left(A_{5}\right)(i)$ holds with $\lambda=3.5213, \epsilon=0.6519$, and $\phi(\kappa+\pi-1)=1$. Since

$$
\frac{1}{\left[\frac{(\beta+n+3)^{(\beta)}}{\Gamma(\beta+1)}+\mathbb{E}_{1}^{*}\left(\frac{\zeta^{(\beta)}}{\Gamma(\beta+1)}+\frac{(\beta+n)^{(\beta-3)}}{\Gamma(\beta-2)}\right)+\mathbb{E}_{2}^{*} \frac{(\beta+n)^{(\beta-1)}}{\Gamma(\beta)}\right]} \approx 0.0137,
$$

if $\mathbb{L}_{G}=0.0014<0.0137$, from Theorem 5, we see that $\Lambda=0.1023<1$. Hence, the solution to Equation (48) is $\mathcal{H U R}$ stable with $\delta_{2}=3.9224$. For illustration, we take $\epsilon=0.6519$ and $\hat{w}(\kappa)=\frac{\kappa^{(3)}}{40}$. We prove that Equation (33) holds. Indeed,

$$
\begin{aligned}
\left|\Delta_{\pi-4}^{\pi} \hat{w}(\kappa)-G(\kappa+\pi-1, \hat{w}(\kappa+\pi-1))\right| \\
\quad=\left|\Delta_{\pi-4}^{\pi} \hat{w}(\kappa)-\frac{1}{700} \sin (\hat{w}(\kappa+\pi-1))-\frac{1}{310}(\kappa+\pi-1)^{(\pi)}\right| \\
=\left|\Delta_{\pi-4}^{\pi}\left(\frac{\kappa^{(3)}}{40}\right)-0.0014 \sin \left[\frac{(\kappa+\pi-1)^{(3)}}{40}\right]-\frac{(\kappa+\pi-1)^{(\pi)}}{310}\right| .
\end{aligned}
$$


Using Lemma 4, Equation (49) becomes

$$
\begin{aligned}
\mid \Delta_{\pi-4}^{\pi} & \hat{w}(\kappa)-G(\kappa+\pi-1, \hat{w}(\kappa+\pi-1)) \mid \\
& =\left|0.1358 \kappa^{(3-\pi)}-0.0014 \sin \left[\frac{\Gamma(\kappa+\pi)}{40 \Gamma(\kappa+\pi-3)}\right]-\frac{\Gamma(\kappa+\pi)}{310 \Gamma(\kappa)}\right| \\
& \leq 0.1358\left[\frac{\Gamma(\kappa+1)}{\Gamma(\kappa-2+\pi)}\right]+0.0014+\frac{\Gamma(\kappa+\pi)}{310 \Gamma(\kappa)} \\
& \leq 0.6519 \leq \epsilon \phi(\kappa+\pi-1), \text { for } \kappa \in \mathbb{N}_{0}^{4} .
\end{aligned}
$$

Furthermore, it is obviously $\mathcal{G} \mathcal{H U} \mathcal{R}$ stable from Remark 3.

\section{Conclusions}

Three-point BCs for a discrete FEBE have been investigated in this research work. For our proposed problem involving a Riemann-Liouville discrete fractional operator, some important conditions for the existence and stability theory have been developed. The required findings have been obtained with the help of fixed-point techniques such as the contraction mapping principle and Brouwer fixed-point theorem. Moreover, some new results for various types of Ulam stability of the proposed three-point BCs for a discrete FEBE have been established with the aid of nonlinear analysis. Some suitable examples have been provided and accompanied with numerical experiment for our obtained solutions for various fractional-order values in a graphical representation in order to study the effectiveness and applicability of our theoretical results. All in all, our results are new and interesting for the elastic beam problem arising from mathematical models of engineering and applied science applications.

Author Contributions: Conceptualization, J.A., R.D. and M.K.A.K.; Formal analysis, J.A., A.G.M.S., R.D. and M.K.A.K.; Investigation, J.A., A.G.M.S. and M.K.A.K.; Methodology, M.K.A.K.; Project administration, J.A.; Supervision, J.A. and M.K.A.K.; Validation, R.D. and M.K.A.K.; Visualization, A.G.M.S.; Writing—original draft, J.A., A.G.M.S., R.D. and M.K.A.K.; Writing-review and editing, J.A., A.G.M.S., R.D. and M.K.A.K. All authors have read and agreed to the published version of the manuscript.

Funding: J. Alzabut would like to thank Prince Sultan University for supporting this work.

Institutional Review Board Statement: Not applicable.

Informed Consent Statement: Not applicable.

Data Availability Statement: Not applicable.

Conflicts of Interest: The authors declare that they have no competing interest.

\section{References}

1. Cianciaruso, F.; Infante, G.; Pietramala, P. Solutions of perturbed hammerstein integral equations with applications. arXiv 2016, arXiv:1602.00976v4.

2. Infante, G.; Pietramala, P. A cantilever equation with nonlinear boundary conditions. Electron. J. Qual. Theory Differ. Equ. 2009, 15, 1-14. [CrossRef]

3. Li, Y. Existence of positive solutions for the cantilever beam equations with fully nonlinear terms. Nonlinear Anal. Real World Appl. 2016, 27, 221-237. [CrossRef]

4. Song, Y. A nonlinear boundary value problem for fourth-order elastic beam equations. Bound. Value Probl. 2014, 191, 1-11. [CrossRef]

5. Li, S.; Zhai, C. New existence and uniqueness results for an elastic beam equation with nonlinear boundary conditions. Bound. Value Probl. 2015, 104, 1-12. [CrossRef]

6. Gupta, C.P. Existence and uniqueness theorems for the bending of an elastic beam equation. Appl. Anal. 1988, 26, 289-304. [CrossRef]

7. Kumar, A.; Chauhan, H.V.S.; Ravichandran, C.; Nisar, K.S.; Baleanu, D. Existence of solutions of non-autonomous fractional differential equations with integral impulse condition. Adv. Differ. Equ. 2020, 434, 1-15. [CrossRef]

8. Ismail, M.; Saeed, U.; Alzabut, J.; Rehman, M. Approximate solutions for fractional boundary value problems via green-cas wavelet method. Mathematics 2019 7, 1164. [CrossRef] 
9. Kilbas, A.A.; Srivastava, H.M.; Trujillo, J.J. Theory and Applications of Fractional Differential Equations; Elsevier Science: Amsterdam, The Netherlands, 2006.

10. Podlubny, I. Fractional Differential Equations; Academic Press: New York, NY, USA, 1999.

11. Benchohra, M.; Litimein, S.; Nieto, J.J. Semilinear fractional differential equations with infinite delay and non-instantaneous impulses. J. Fixed Point Theory Appl. 2019, 21, 1-21. [CrossRef]

12. Almeida, R. What is the best fractional derivative to fit data? Appl. Anal. Discrete Math. 2017, 11, 358-368. [CrossRef]

13. Qureshi, S.; Yusuf, A. Fractional derivatives applied to MSEIR problems: Comparative study with real world data. Eur. Phys. J. Plus 2019, 134, 1-17. [CrossRef]

14. Matar, M.M.; Abbas, M.I.; Alzabut, J.; Kaabar, M.K.A.; Etemad, S.; Rezapour, S. Investigation of the p-Laplacian nonperiodic nonlinear boundary value problem via generalized Caputo fractional derivatives. Adv. Differ. Equ. 2021, 68, 1-18.

15. Pratap, A.; Raja, V.; Alzabut, J.; Dianavinnarasi, J.; Cao, J.; Rajchakit, G. Finite-time Mittag-Leffler stability of fractional-order quaternion-valued memristive neural networks with impulses. Neural Process Lett. 2020, 51, 1485-1526. [CrossRef]

16. Wu, R.C.; Hei, X.D.; Chen, L.P. Finite-time stability of fractional-order neural networks with delay. Commun. Theor. Phys. 2013, 60, 189-193. [CrossRef]

17. Trigeassou, J.C.; Maamri, N.; Sabatier, J.; Oustaloup, A. A Lyapunov approach to the stability of fractional differential equations. Signal Process 2011, 91, 437-445. [CrossRef]

18. Stamova, I. Mittag-Leffler stability of impulsive differential equations of fractional order. Q. Appl. Math. 2015, 73, 525-535. [CrossRef]

19. Lijun, G.; Wang, D.; Wang, G. Further results on exponential stability for impulsive switched nonlinear time-delay systems with delayed impulse effects. Appl. Math. Comput. 2015, 268, 186-200.

20. Hyers, D. On the stability of the linear functional equation. Proc. Natl. Acad Sci. USA 1941, 27, 222-224. [CrossRef] [PubMed]

21. Obloza, M. Hyers stability of the linear differential equation. Rocznik Nauk-Dydakt Prace Mat. 1993, 13, 259-270.

22. Ulam, S.M. Problems in Modern Mathematics; Wiley: New York, NY, USA, 1940.

23. Ahmad, M.; Zada, A.; Alzabut, J. Stability analysis of a nonlinear coupled implicit switched singular fractional differential system with p-Laplacian. Adv. Differ. Equ. 2019, 436, 1-22. [CrossRef]

24. Zada, A.; Alzabut, J.; Waheed, H.; Loan-Lucian, P. Ulam-Hyers stability of impulsive integrodifferential equations with RiemannLiouville boundary conditions. Adv. Differ. Equ. 2020, 1, 1-50. [CrossRef]

25. Iswarya, M.; Raja, R.; Rajchakit, G.; Cao, J.; Alzabut, J.; Huang, C. Existence, uniqueness and exponential stability of periodic solution for discrete-time delayed BAM neural networks based on coincidence degree theory and graph theoretic method. Mathematics 2019, 7, 1055. [CrossRef]

26. Guo, Y.; Shu, X.; Li, Y.; Xu, F. The existence and Hyers-Ulam stability of solution for an impulsive Riemann-Liouville fractional neutral functional stochastic differential equation with infinite delay of order $1<\beta<2$. Bound. Value Prob. 2019, 59. [CrossRef]

27. Ahmad, M.; Zada, A.; Alzabut, J. Hyres-Ulam Stability of Coupled System of Fractional Differential Equations of HilferHadamard Type. Demonstr. Math. 2019, 52, 283-295. [CrossRef]

28. Salem, A.; Alzahrani, F.; Almaghamsi, L. Fractional Langevin equations with nonlocal integral boundary conditions. Mathematics 2019, 7, 402. [CrossRef]

29. Abdeljawad, T. On Riemann and Caputo fractional differences. Comput. Math. Appl. 2011, 62, 1602-1611. [CrossRef]

30. Atici, F.M.; Eloe, P.W. A transform method in discrete fractional calculus. Int. J. Difference Equ. 2017, 2, $165-176$.

31. Atici, F.M.; Eloe, P.W. Initial value problems in discrete fractional calculus. Proc. Am. Math. Soc. 2009, 137, 981-989. [CrossRef]

32. Miller, K.S.; Ross, B. Fractional difference calculus. In Proceedings of the International Symposium on Univalent Functions, Fractional Calculus and their Applications; Nihon University: Koriyama, Japan, 1988; pp. 139-152.

33. Goodrich, C.S.; Peterson, A.C. Discrete Fractional Calculus; Springer: New York, NY, USA, 2015.

34. Atici, F.M.; Eloe, P.W. Two-point boundary value problems for finite fractional difference equations. J. Differ. Equ. Appl. 2011, 17, 445-456. [CrossRef]

35. Alzabut, J.; Abdeljawad, T.; Baleanu, D. Nonlinear delay fractional difference equations with applications on discrete fractional Lotka-Volterra competition model. J. Comput. Anal. Appl. 2018, 25, 889-898.

36. Chen, F.; Zhou, Y. Existence and Ulam Stability of Solutions for Discrete Fractional Boundary Value Problem. Discret. Dyn. Nat. Soc. 2013, 1-7. [CrossRef]

37. Chen, H.; Jin, Z.; Kang, S. Existence of positive solution for Caputo fractional difference equation. Adv. Differ. Equ. 2015, 44, 1-12. [CrossRef]

38. Chen, C.; Bohner, M.; Jia, B. Ulam-Hyers stability of Caputo fractional difference equations. Math. Meth. Appl. Sci. 2019, 42, 7461-7470. [CrossRef]

39. Chen, C.; Bohner, M.; Jia, B. Method of upper and lower solutions for nonlinear Caputo fractional difference equations and its applications. Fract. Calc. Appl. Anal. 2019, 22, 1307-1320. [CrossRef]

40. Chen, C.; Bohner, M.; Jia, B. Existence and uniqueness of solutions for nonlinear Caputo fractional difference equations. Turk. J. Math. 2020, 44, 857-869. [CrossRef]

41. Goodrich, C.S. Solutions to a discrete right-focal fractional boundary value problem. Int. J. Differ. Equ. 2010, 5, 195-216.

42. Goodrich, C.S. Existence and uniqueness of solutions to a fractional difference equation with nonlocal conditions. Comput. Math. Appl. 2011, 61, 191-202. [CrossRef] 
43. Rehman, M.; Iqbal, F.; Seemab, A. On existence of positive solutions for a class of discrete fractional boundary value problems. Positivity 2017, 21, 1173-1187. [CrossRef]

44. Selvam, A.G.M.; Dhineshbabu, R. Uniqueness of solutions of a discrete fractional order boundary value problem. AIP Conf. Proc. 2019, 2095, 1-7.

45. Selvam, A.G.M.; Alzabut, J.; Dhineshbabu, R.; Rashid, S.; Rehman, M. Discrete fractional order two-point boundary value problem with some relevant physical applications. J. Inequal. Appl. 2020, 221, 1-19.

46. Selvam, A.G.M.; Dhineshbabu, R. Ulam stability results for boundary value problem of fractional difference equations. Adv. Math. Sci. J. 2020, 9, 219-230. [CrossRef]

47. Selvam, A.G.M.; Dhineshbabu, R. Existence and uniqueness of solutions for a discrete fractional boundary value problem. Int. J. Appl. Math. 2020, 33, 283-295. 